\title{
SOLVING A STRUCTURED QUADRATIC EIGENVALUE PROBLEM BY A STRUCTURE-PRESERVING DOUBLING ALGORITHM*
}

\author{
CHUN-HUA GUO ${ }^{\dagger}$ AND WEN-WEI LIN ${ }^{\ddagger}$
}

\begin{abstract}
In studying the vibration of fast trains, we encounter a palindromic quadratic eigenvalue problem $(\mathrm{QEP})\left(\lambda^{2} A^{T}+\lambda Q+A\right) z=0$, where $A, Q \in \mathbb{C}^{n \times n}$ and $Q^{T}=Q$. Moreover, the matrix $Q$ is block tridiagonal and block Toeplitz, and the matrix $A$ has only one nonzero block in the upperright corner. So most of the eigenvalues of the QEP are zero or infinity. In a linearization approach, one typically starts with deflating these known eigenvalues, for the sake of efficiency. However, this initial deflation process involves the inverses of two potentially ill-conditioned matrices. As a result, large error might be introduced into the data for the reduced problem. In this paper we propose using the solvent approach directly on the original QEP, without any deflation process. We apply a structure-preserving doubling algorithm to compute the stabilizing solution of the matrix equation $X+A^{T} X^{-1} A=Q$, whose existence is guaranteed by a result on the Wiener-Hopf factorization of rational matrix functions associated with semi-infinite block Toeplitz matrices and a generalization of Bendixson's theorem to bounded linear operators on Hilbert spaces. The doubling algorithm is shown to be well defined and quadratically convergent. The complexity of the doubling algorithm is drastically reduced by using the Sherman-Morrison-Woodbury formula and the special structures of the problem. Once the stabilizing solution is obtained, all nonzero finite eigenvalues of the QEP can be found efficiently and with the automatic reciprocal relationship, while the known eigenvalues at zero or infinity remain intact.
\end{abstract}

Key words. palindromic quadratic eigenvalue problem, nonlinear matrix equation, stabilizing solution, structure-preserving, doubling algorithm

AMS subject classifications. 15A24, 65F15, 65F 30

1. Introduction. In this paper we consider a quadratic eigenvalue problem (QEP) occurring in the vibration analysis of rail tracks under excitation arising from high speed trains $[14,15,17]$. This problem has provided much of the motivation for the study of palindromic polynomial eigenvalue problems in [22] and subsequent papers $[5,16,18,19,20,23]$. Yet the problem itself has not been solved satisfactorily.

The standard approach for solving a QEP is to use a proper linearization and solve a generalized eigenvalue problem of twice the size. Another approach for solving a QEP is through finding a solution (called a solvent) of a related matrix equation. This solvent approach has been explored in [6] and more recently in [13] and [26]. The difficulty associated with the solvent approach is obvious. It is possible that the related matrix equation does not have a solvent. Even if a solvent exists, the computation of a solvent can still be a difficult task. As a result, the solvent approach can outperform the linearization approach only for special types of QEPs $[9,11]$.

So far every method for the special QEP here starts with the linearization approach. For the sake of efficiency, a deflation process is used in the beginning. This initial deflation process involves the sucessive application of the inverses of two potentially ill-conditioned matrices (see [5] for example). As a result, large error might be introduced into the data for the reduced problem. Several methods have been

\footnotetext{
*Version of July 29, 2010.

${ }^{\dagger}$ Department of Mathematics and Statistics, University of Regina, Regina, SK S4S 0A2, Canada (chguo@math.uregina.ca). The work of this author was supported in part by a grant from the Natural Sciences and Engineering Research Council of Canada and by the National Center for Theoretical Sciences in Taiwan.

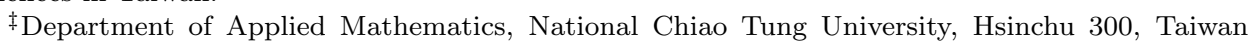
(wwlin@math.nctu.edu.tw). The work of this author was partially supported by the National Science Council and the National Center for Theoretical Sciences in Taiwan.
} 
proposed recently to solve the reduced QEP. In particular, the solvent approach is used in [5]. However, some major issues associated with the solvent approach remain unsolved in [5]. Another efficient method is proposed and compared to two other methods in [16]. These methods continue to use the linearization approach for the reduced QEP. The computational work for all these different methods are dominated by that of the same deflation process. The accuracy of the computed solution is thus the main issue here.

In this paper we will see that the QEP arising in the study of high speed trains is very amenable for the solvent approach, if used directly on the original QEP, without any deflation process. At first sight, the solvent approach applied to the original QEP would also be very expensive. In this paper we will show that the solvent approach can be implemented to have a complexity roughly the same as that for other efficient methods using the linearization approach and the initial deflation process. Numerical experiments show that our solvent approach, applied to the original QEP, produces better accuracy in the computed results.

2. The quadratic eigenvalue problem. The vibration analysis of rail tracks can be performed through a finite element model, in which we generate [5] two real symmetric matrices $M$ and $K$ of the form

$$
\begin{aligned}
& M=\left[\begin{array}{cccccc}
M_{0} & M_{1}^{T} & 0 & \ldots & 0 & M_{1} \\
M_{1} & M_{0} & M_{1}^{T} & 0 & & 0 \\
0 & \ddots & \ddots & \ddots & \ddots & \vdots \\
\vdots & 0 & \ddots & \ddots & \ddots & 0 \\
0 & & \ddots & M_{1} & M_{0} & M_{1}^{T} \\
M_{1}^{T} & 0 & \cdots & 0 & M_{1} & M_{0}
\end{array}\right]_{m \times m} \\
& K=\left[\begin{array}{cccccc}
K_{0} & K_{1}^{T} & 0 & \cdots & 0 & K_{1} \\
K_{1} & K_{0} & K_{1}^{T} & 0 & & 0 \\
0 & \ddots & \ddots & \ddots & \ddots & \vdots \\
\vdots & 0 & \ddots & \ddots & \ddots & 0 \\
0 & & \ddots & K_{1} & K_{0} & K_{1}^{T} \\
K_{1}^{T} & 0 & \cdots & 0 & K_{1} & K_{0}
\end{array}\right]_{m \times m}
\end{aligned}
$$

where each block in $M$ and $K$ is $q \times q$. So $M, K \in \mathbb{R}^{n \times n}$ with $n=m q$. The matrices $M$ and $K$ are thus block Toeplitz (actually block circulant). This special structure is not used in [5] and the notation used there for $M$ and $K$ is more general. A matrix $D$ (the damping matrix) is then taken to be a positive linear combination of $M$ and $K$. That is, $D=c_{1} M+c_{2} K$ with $c_{1}, c_{2}>0$. So $D$ has the same structure as $M$ and $K$. We write $M=M_{t}+M_{c}+M_{c}^{T}$, where $M_{t}$ is the block tridiagonal part of $M$, and $M_{c}$ is the matrix with $M_{1}$ in the upper-right corner and zero blocks elsewhere. Similarly, we have $K=K_{t}+K_{c}+K_{c}^{T}$ and $D=D_{t}+D_{c}+D_{c}^{T}$. We also denote by $\omega>0$ the frequency of the external excitation force.

For the vibration anaysis, one needs to solve the palindromic QEP [5]

$$
P(\lambda) z=0, \quad z \neq 0
$$


with

$$
P(\lambda)=\lambda^{2} A^{T}+\lambda Q+A,
$$

where

$$
Q=K_{t}+i \omega D_{t}-\omega^{2} M_{t}
$$

with $i=\sqrt{-1}$ (so $\left.Q^{T}=Q\right)$, and

$$
A=K_{c}+i \omega D_{c}-\omega^{2} M_{c} .
$$

The set of eigenvalues of the quadratic $P(\lambda)$ demonstrates a "symplectic" behaviour (i.e., a symmetry with respect to the unit circle, which is denoted by $\mathbb{T}$ throughout this paper). More precisely, a number $\xi$ is an eigenvalue of the quadratic $P(\lambda)$ if and only if $\xi^{-1}$ is so, and they have the same partial multiplicities (see [22, Theorem 2.2]).

Let $\ell_{2}$ be the usual Hilbert space of all square summable sequence of complex numbers, and let $\ell_{2}^{q}$ be the Cartesian product of $q$ copies of $\ell_{2}$. The infinite matrices

$$
T_{M}=\left[\begin{array}{cccc}
M_{0} & M_{1}^{T} & & \\
M_{1} & M_{0} & M_{1}^{T} & \\
& M_{1} & M_{0} & \ddots \\
& & \ddots & \ddots
\end{array}\right], \quad T_{K}=\left[\begin{array}{cccc}
K_{0} & K_{1}^{T} & & \\
K_{1} & K_{0} & K_{1}^{T} & \\
& K_{1} & K_{0} & \ddots \\
& & \ddots & \ddots
\end{array}\right]
$$

are then seen to be in $\mathcal{B}\left(\ell_{2}^{q}\right)$, the set of all bounded linear operators on $\ell_{2}^{q}$. They are also self-adjoint operators in $\mathcal{B}\left(\ell_{2}^{q}\right)$. It is well known that the spectrum of a self-adjoint operator is real [27].

By the way the matrices $M$ and $K$ are generated in the finite element model, we know that $T_{K}$ is positive semidefinite, written $T_{K} \geq 0$, in the sense that $\left\langle T_{K} f, f\right\rangle \geq 0$ for all $f \in \ell_{2}^{q}$. We also know that $T_{M} \geq \epsilon I$ (i.e., $T_{M}-\epsilon I \geq 0$ ) for the identity operator $I$ and some $\epsilon>0$. These properties on $T_{K}$ and $T_{M}$ can also be verified independently (in case significant errors are introduced in setting up the matrices $M_{0}, M_{1}, K_{0}, K_{1}$ ). As noted in [7], $T_{K} \geq 0$ if and only if $\psi_{K}(\lambda)=K_{0}+\lambda K_{1}+\lambda^{-1} K_{1}^{T}$ is positive semidefinite on $\mathbb{T}$. So $T_{M} \geq \epsilon I$ for some $\epsilon>0$ if and only if $\psi_{M}(\lambda)=M_{0}+\lambda M_{1}+\lambda^{-1} M_{1}^{T}$ is positive definite on $\mathbb{T}$. The latter holds if and only if the matrix equation

$$
X+M_{1}^{T} X^{-1} M_{1}=M_{0}
$$

has a positive definite solution $X$ with $\rho\left(X^{-1} M_{1}\right)<1$ (see [7]), where $\rho(\cdot)$ denotes the spectral radius. The equation (2.8), where $M_{0}$ is symmetric positive definite, has been well studied (see $[4,7,9,10,21,25,30]$ ). In particular, instead of checking $\psi_{M}(\lambda)$ is positive definite on $\mathbb{T}$, one can attempt to find the maximal positive definite solution of the equation (2.8) by the cyclic reduction method in [25] or the doubling algorithm in [21]. These methods are very efficient, and the computational work involved is only a small fraction of that for solving the QEP, which involves $m q \times m q$ matrices while the matrices in (2.8) are $q \times q$. Recall that $D=c_{1} M+c_{2} K$ for $c_{1}, c_{2}>0$. So $T_{D} \geq c_{1} \epsilon I$, where

$$
T_{D}=\left[\begin{array}{cccc}
D_{0} & D_{1}^{T} & & \\
D_{1} & D_{0} & D_{1}^{T} & \\
& D_{1} & D_{0} & \ddots \\
& & \ddots & \ddots
\end{array}\right], \quad D_{0}=c_{1} M_{0}+c_{2} K_{0}, \quad D_{1}=c_{1} M_{1}+c_{2} K_{1} .
$$


From now on we assume that $T_{D} \geq \eta I$ for some $\eta>0$, which can be verified as we have described for $T_{M}$.

3. Theoretical results for the solvent approach. We first show that the QEP does not have any eigenvalues on $\mathbb{T}$. The following result, due essentially to Bendixson [2], can be found in [28].

Lemma 3.1. (Bendixson's theorem) Let $X$ and $Y$ be any $k \times k$ Hermitian matrices. Suppose that the eigenvalues of $X$ are $\lambda_{1} \leq \lambda_{2} \leq \cdots \leq \lambda_{k}$ and the eigenvalues of $Y$ are $\mu_{1} \leq \mu_{2} \leq \cdots \leq \mu_{k}$. Then the eigenvalues of $X+i Y$ are contained in the rectangle $\left[\lambda_{1}, \lambda_{k}\right] \times\left[\mu_{1}, \mu_{k}\right]$ in the complex plane.

Theorem 3.2. The quadratic $P(\lambda)$ in (2.4) has no eigenvalues on $\mathbb{T}$.

Proof. The quadratic $P(\lambda)$ has eigenvalues on $\mathbb{T}$ if and only if $\operatorname{det} P(\lambda)=0$ for some $\lambda \in \mathbb{T}$, or equivalently $\operatorname{det}\left(\lambda A^{T}+\lambda^{-1} A+Q\right)=0$ for some $\lambda \in \mathbb{T}$. This is impossible since we can show that for each fixed $\lambda \in \mathbb{T}$ all eigenvalues of the matrix $\lambda A^{T}+\lambda^{-1} A+Q$ have positive imaginary parts. In fact, $\lambda A^{T}+\lambda^{-1} A+Q=$ $X(\lambda)+i Y(\lambda)$ with Hermitian matrices

$$
\begin{gathered}
X(\lambda)=K_{t}-\omega^{2} M_{t}+\lambda^{-1}\left(K_{c}-\omega^{2} M_{c}\right)+\lambda\left(K_{c}-\omega^{2} M_{c}\right)^{T} \\
Y(\lambda)=\omega\left(D_{t}+\lambda^{-1} D_{c}+\lambda D_{c}^{T}\right) .
\end{gathered}
$$

By Bendixson's theorem, we only need to show that $Y(\lambda)>0$ on $\mathbb{T}$, or equivalently $\widehat{T}_{D} \geq \epsilon I$ for some $\epsilon>0$, where

$$
\widehat{T}_{D}=\left[\begin{array}{cccc}
D_{t} & D_{c}^{T} & & \\
D_{c} & D_{t} & D_{c}^{T} & \\
& D_{c} & D_{t} & \ddots \\
& & \ddots & \ddots
\end{array}\right] .
$$

The latter is true since $\widehat{T}_{D}$ is precisely (a partition of) $T_{D}$ in (2.9).

We now consider the matrix equation

$$
X+A^{T} X^{-1} A=Q,
$$

where $Q$ and $A$ are given in (2.5) and (2.6). Suppose $X$ is a solution of (3.2). Then we have the factorization for the quadratic $P(\lambda)$ in (2.4):

$$
\lambda^{2} A^{T}+\lambda Q+A=\left(\lambda A^{T}+X\right) X^{-1}(\lambda X+A) .
$$

So the eigenvalues of the quadratic $P(\lambda)$ are a collection of the eigenvalues of the pencils $\lambda A^{T}+X$ and $\lambda X+A$. We have already shown that $P(\lambda)$ has no eigenvalues on $\mathbb{T}$. Suppose a solution $X$ of (3.2) can be found such that the eigenvalues of the pencil $\lambda X+A$ (or equivalently the eigenvalues of the matrix $-X^{-1} A$ ) are inside $\mathbb{T}$. Then the remaining eigenvalues of $P(\lambda)$ are obtained by taking the reciprocals of these eigenvalues. Such a solution $X$ is called a stabilizing solution of (3.2). In this process, the known eigenvalues of $P(\lambda)$ at zero or infinity remain intact, regardless of the accuracy of the computed $X$.

There are two advantages of the solvent approach over the linearization approach. First, in the linearization approach, a deflation procedure is used for the sake of efficiency, which involves the inverses of two potentially ill-conditioned matrices. When 
the QEP is reduced to a smaller QEP (even if in a structure-preserving manner), the input data obtained in the smaller QEP could be significantly different from the true data. In the solvent approach, the ill-conditioning of those matrices may also affect the accuracy of the solution $X$ computed by some efficient method, but we can always use Newton's method as a correction method afterwards, as in [10]. Second, in the linearization approach, the eigenvalues of the smaller QEP range in modulus from $\epsilon$ to $\epsilon^{-1}$, where $\epsilon$ is close to 0 , while in the solvent approach the eigenvalues of $\lambda X+A$ range in modulus from $\epsilon$ to 1 . The situation in the solvent approach is easier to handle, and the symplectic structure of the eigenvalues of $P(\lambda)$ is preserved automatically.

The success of the solvent approach hinges on the existence of a stabilizing solution of (3.2) and an efficient method for its computation. In this section we prove the existence of a stabilizing solution. In the next section we show that a doubling algorithm can be used to compute it efficiently.

We start with a generalization of Bendixson's theorem to bounded linear operators in Hilbert spaces. It seems that such a generalization has not been given before, although special cases of this are being proved in recent literature.

Lemma 3.3. (generalization of Bendixson's theorem) Let $B$ and $C$ be self-adjoint bounded linear operators on a Hilbert space. Suppose that the spectrum of $B$ is contained in $\left[u_{1}, u_{2}\right]$ and the spectrum of $C$ is contained in $\left[v_{1}, v_{2}\right]$. Then the spectrum of $B+i C$ is contained in the rectangle $\left[u_{1}, u_{2}\right] \times\left[v_{1}, v_{2}\right]$ in the complex plane.

Proof. Some special cases have been proved in the literature. For example, it is proved in [1] (see Corollary 4 there) that $B+i C$ is invertible if $B$ is invertible and positive definite (or equivalently [27, Section 7.4, Corollary 2] if $\min \sigma(B) \geq \epsilon$ for some $\epsilon>0$ ). Also, it is proved in [12] (see the proof of Lemma 3.1 there) that $B+i C$ is invertible if $C \geq \epsilon I$ for some $\epsilon>0$ (or equivalently [27, Section 7.4, Corollary 2] if $\min \sigma(C) \geq \epsilon)$. Note that the result in [12] follows from the result in [1] by a multiplication with $i$. The general statement in Lemma 3.3 can also be proved quickly using the special case proved in [1]. We only need to prove that each point $a+b i$ in the spectrum of $B+i C$ satisfies $a \geq u_{1}$ (the rest can be proved by multiplying $B+i C$ with -1 or $i$ ). We may assume $u_{1}>0$ by shifting $B$ to $B+\eta I$ for some $\eta>0$. Since $\sigma(B+i C)$ is a compact set [27, Theorem 5.14], the distance between the imaginary axis and $\sigma(B+i C)$ is attained for a point $a^{*}+b^{*} i$ in $\sigma(B+i C)$. We need to show $a^{*} \geq u_{1}$. Suppose $a^{*}<u_{1}$. Then $B-a^{*} I \geq\left(u_{1}-a^{*}\right) I$ with $u_{1}-a^{*}>0$ and thus $(B+i C)-\left(a^{*}+b^{*} i\right) I=\left(B-a^{*} I\right)+i\left(C-b^{*} I\right)$ is invertible by [1, Corollary 4]. This is a contradiction since $a^{*}+b^{*} i$ is in $\sigma(B+i C)$.

To prove the existence of a stabilizing solution of (3.2), we consider the semiinfinite block Toeplitz matrix

$$
T=\left[\begin{array}{cccc}
Q & A^{T} & & \\
A & Q & A^{T} & \\
& A & Q & \ddots \\
& & \ddots & \ddots
\end{array}\right]
$$

Associated with $T$ is the rational matrix function $\phi(\lambda)=\lambda A+Q+\lambda^{-1} A^{T}$. It is clear that $T$ is in $\mathcal{B}\left(\ell_{2}^{n}\right)$ and we will show that $T$ is invertible. 
By (2.5) and (2.6) we have $T=B+i C$ with

$$
B=\left[\begin{array}{cccc}
B_{t} & B_{c}^{T} & & \\
B_{c} & B_{t} & B_{c}^{T} & \\
& B_{c} & B_{t} & \ddots \\
& & \ddots & \ddots
\end{array}\right],
$$

where $B_{t}=K_{t}-\omega^{2} M_{t}$ and $B_{c}=K_{c}-\omega^{2} M_{c}$, and $C=\omega \widehat{T}_{D}$, where $\widehat{T}_{D}$ is given in (3.1). Note that $B$ and $C$ are self-adjoint operators in $\mathcal{B}\left(\ell_{2}^{n}\right)$. Since $\widehat{T}_{D}$ is a partition of $T_{D}$ and $T_{D} \geq \eta I$ for some $\eta>0$, we have $C \geq \omega \eta I$ and thus $\min \sigma(C) \geq \omega \eta$. By Lemma $3.3,0 \notin \sigma(B+i C)$. So $T=B+i C$ is invertible.

We can now prove the following result.

THEOREM 3.4. The equation (3.2) has a unique stabilizing solution, and the solution is complex symmetric. Moreover, the dual equation of (3.2)

$$
\widehat{X}+A \widehat{X}^{-1} A^{T}=Q
$$

also has a unique stabilizing solution and the solution is complex symmetric.

Proof. Since $T$ is invertible, we know from a result on linear operators (see [8, Chapter XXIV, Theorem 4.1] and [24]) that $\phi(\lambda)$ has the so-called Wiener-Hopf factorization

$$
\phi(\lambda)=\left(I-\lambda^{-1} L\right) D(I-\lambda U)
$$

with $D$ invertible, $\rho(L)<1$ and $\rho(U)<1$. From (3.5) we see that

$$
A=-D U, \quad A^{T}=-L D, \quad Q=D+L D U .
$$

Thus

$$
D+A^{T} D^{-1} A=Q
$$

with $\rho\left(D^{-1} A\right)<1$ and $\rho\left(A^{T} D^{-1}\right)<1$. So $D$ is a stabilizing solution of the equation (3.2). We will see in the next section that the pencil $N_{0}-\lambda L_{0}$ defined by (4.1) has exactly $n$ eigenvalues inside $\mathbb{T}$, and that for any stabilizing solution $X_{s}$ of (3.2) the column space of $\left[\begin{array}{ll}I & X_{s}^{T}\end{array}\right]^{T}$ is, by (4.3), the (necessarily unique) deflating subspace of the pencil $N_{0}-\lambda L_{0}$ corresponding to its $n$ eigenvalues inside $\mathbb{T}$. It follows that (3.2) has exactly one stabilizing solution. Now, taking transpose in (3.6) gives $D^{T}+$ $A^{T}\left(D^{T}\right)^{-1} A=Q$. Note that $\rho\left(\left(D^{T}\right)^{-1} A\right)=\rho\left(A^{T} D^{-1}\right)<1$. So $D^{T}$ is also a stabilizing solution of (3.2). The uniqueness of stabilizing solutions implies that $D^{T}=$ $D$.

The statements about the dual equation can be proved in a similar way. The only difference is that we now need to show that the self-adjoint operator in $\mathcal{B}\left(\ell_{2}^{n}\right)$

$$
\widetilde{T}_{D}=\left[\begin{array}{cccc}
D_{t} & D_{c} & & \\
D_{c}^{T} & D_{t} & D_{c} & \\
& D_{c}^{T} & D_{t} & \ddots \\
& & \ddots & \ddots
\end{array}\right]
$$

is such that $\widetilde{T}_{D} \geq \epsilon I$ for some $\epsilon>0$. This is true since $\widetilde{T}_{D}$ is related to $\widehat{T}_{D}$ in (3.1) by

$$
\widetilde{T}_{D}=W \widehat{T}_{D} W,
$$


where

$$
W=\left[\begin{array}{ccc}
V & & \\
& V & \\
& & \ddots
\end{array}\right], \quad V=\left[\begin{array}{lll} 
& & I_{q} \\
I_{q} & &
\end{array}\right]_{m \times m},
$$

and $I_{q}$ is the $q \times q$ identity matrix. Thus for any $f \in \ell_{2}^{n},\left\langle\widetilde{T}_{D} f, f\right\rangle=\left\langle\widehat{T}_{D}(W f), W f\right\rangle \geq$ $\eta\|W f\|^{2}=\eta\|f\|^{2}$. So $\widetilde{T}_{D} \geq \eta I$.

4. Computation of the stabilizing solution. A doubling algorithm has been studied in [21] for the equation (3.2) with a real $A$ and a real symmetric positive definite $Q$. In our case, $A$ is complex and $Q$ is complex symmetric. However, the more general presentation in [4] can be used directly.

Let

$$
N_{0}=\left[\begin{array}{cc}
A & 0 \\
Q & -I
\end{array}\right], \quad L_{0}=\left[\begin{array}{cc}
0 & I \\
A^{T} & 0
\end{array}\right] .
$$

Then the pencil $N_{0}-\lambda L_{0}$ is a linearization of the $T$-palindromic polynomial $\lambda^{2} A^{T}-$ $\lambda Q+A$. It is easy to verify that the pencil $N_{0}-\lambda L_{0}$ is $T$-symplectic, i.e.,

$$
N_{0} J N_{0}^{T}=L_{0} J L_{0}^{T} \text { for } J=\left[\begin{array}{cc}
0 & I \\
-I & 0
\end{array}\right] .
$$

We can define the sequences $\left\{N_{k}\right\}$ and $\left\{L_{k}\right\}$, where

$$
N_{k}=\left[\begin{array}{cc}
A_{k} & 0 \\
Q_{k} & -I
\end{array}\right], \quad L_{k}=\left[\begin{array}{cc}
-P_{k} & I \\
A_{k}^{T} & 0
\end{array}\right]
$$

by the following doubling algorithm [4] if no breakdown occurs.

Algorithm 4.1. Let $A_{0}=A, Q_{0}=Q, P_{0}=0$.

For $k=0,1, \ldots$, compute

$$
\begin{aligned}
A_{k+1} & =A_{k}\left(Q_{k}-P_{k}\right)^{-1} A_{k}, \\
Q_{k+1} & =Q_{k}-A_{k}^{T}\left(Q_{k}-P_{k}\right)^{-1} A_{k}, \\
P_{k+1} & =P_{k}+A_{k}\left(Q_{k}-P_{k}\right)^{-1} A_{k}^{T} .
\end{aligned}
$$

We now show that this algorithm will not break down, and $Q_{k}$ converges quadratically to the stabilizing solution of (3.2).

TheOrem 4.1. Let $A$ and $Q$ be given by (2.6) and (2.5). Let $X_{s}$ be the stabilizing solution of (3.2) and $\widehat{X}_{s}$ be the stabilizing solution of the dual equation (3.4). Then

(a) The sequences $\left\{A_{k}\right\},\left\{Q_{k}\right\},\left\{P_{k}\right\}$ in Algorithm 4.1 are well-defined, and $Q_{k}$ and $P_{k}$ are complex symmetric.

(b) $Q_{k}$ converges to $X_{s}$ quadratically, $A_{k}$ converges to 0 quadratically, $Q-P_{k}$ converges to $\widehat{X}_{s}$ quadratically, with

$$
\begin{gathered}
\limsup _{k \rightarrow \infty} \sqrt[2^{k}]{\left\|Q_{k}-X_{s}\right\|} \leq\left(\rho\left(X_{s}^{-1} A\right)\right)^{2}, \quad \limsup _{k \rightarrow \infty} \sqrt[2^{k}]{\left\|A_{k}\right\|} \leq \rho\left(X_{s}^{-1} A\right), \\
\limsup _{k \rightarrow \infty} \sqrt[2^{k}]{\left\|Q-P_{k}-\widehat{X}_{s}\right\|} \leq\left(\rho\left(X_{s}^{-1} A\right)\right)^{2},
\end{gathered}
$$

where $\|\cdot\|$ is any matrix norm. 
Proof. Let $T_{k}$ be the leading principal block $k \times k$ submatrix of $T$ in (3.3) and write $T_{k}=B_{k}+i C_{k}$, where $B_{k}$ and $C_{k}$ are Hermitian. So

$$
C_{k}=\omega\left[\begin{array}{cccc}
D_{t} & D_{c}^{T} & & \\
D_{c} & D_{t} & \ddots & \\
& \ddots & \ddots & D_{c}^{T} \\
& & D_{c} & D_{t}
\end{array}\right]_{k \times k}
$$

Since $T_{D} \geq \eta I$, we have $\left\langle T_{D} f, f\right\rangle \geq \eta\|f\|^{2}$ for all $f \in \ell_{2}^{q}$. Taking $f=\left[\begin{array}{l}g \\ 0\end{array}\right]$ with $g \in \mathbb{C}^{k m q}$, we know that for all $g \in \mathbb{C}^{k m q}$,

$$
\left\langle C_{k} g, g\right\rangle=\omega\left\langle T_{D} f, f\right\rangle \geq \omega \eta\|f\|^{2}=\omega \eta\|g\|^{2} .
$$

Thus $C_{k}$ is positive definite for each $k \geq 1$. It then follows from Bendixson's theorem that $T_{k}$ is invertible for each $k \geq 1$.

Let $W_{k}=Q_{k}-P_{k}$ in Algorithm 4.1. Then the sequence $\left\{W_{k}\right\}$ satisfies

$$
W_{k+1}=W_{k}-A_{k}^{T} W_{k}^{-1} A_{k}-A_{k} W_{k}^{-1} A_{k}^{T}, \quad W_{0}=Q .
$$

It follows from [3, Theorem 13, see also equation (9)] that $W_{k}$ is nonsingular for each $k \geq 0$. The sequences $\left\{A_{k}\right\},\left\{Q_{k}\right\},\left\{P_{k}\right\}$ are then well-defined. It is easy to see by induction that $Q_{k}$ and $P_{k}$ are complex symmetric since $Q$ is complex symmetric. This proves (a).

To prove (b), we start with the easily verified relation

$$
N_{0}\left[\begin{array}{c}
I \\
X_{s}
\end{array}\right]=L_{0}\left[\begin{array}{c}
I \\
X_{s}
\end{array}\right] X_{s}^{-1} A
$$

From the discussions in [4] we have for each $k \geq 0$

$$
N_{k}\left[\begin{array}{c}
I \\
X_{s}
\end{array}\right]=L_{k}\left[\begin{array}{c}
I \\
X_{s}
\end{array}\right]\left(X_{s}^{-1} A\right)^{2^{k}}
$$

Substituting (4.2) into (4.4) yields

$$
A_{k}=\left(X_{s}-P_{k}\right)\left(X_{s}^{-1} A\right)^{2^{k}}, \quad Q_{k}-X_{s}=A_{k}^{T}\left(X_{s}^{-1} A\right)^{2^{k}} .
$$

Similarly we have

$$
\widehat{N}_{0}\left[\begin{array}{c}
I \\
\widehat{X}_{s}
\end{array}\right]=\widehat{L}_{0}\left[\begin{array}{c}
I \\
\widehat{X}_{s}
\end{array}\right] \widehat{X}_{s}^{-1} A^{T}
$$

where

$$
\widehat{N}_{0}=\left[\begin{array}{cc}
A^{T} & 0 \\
Q & -I
\end{array}\right], \quad \widehat{L}_{0}=\left[\begin{array}{cc}
0 & I \\
A & 0
\end{array}\right] .
$$

The pencil $\widehat{N}_{0}-\lambda \widehat{L}_{0}$ is a linearization of $\lambda^{2} A-\lambda Q+A^{T}$, which has the same eigenvalues as $\lambda^{2} A^{T}-\lambda Q+A$. It follows that $\widehat{X}_{s}^{-1} A^{T}$ and $X_{s}^{-1} A$ have the same eigenvalues, and thus $\rho\left(\widehat{X}_{s}^{-1} A^{T}\right)=\rho\left(X_{s}^{-1} A\right)$. For each $k \geq 0$ we now have

$$
\widehat{N}_{k}\left[\begin{array}{c}
I \\
\widehat{X}_{s}
\end{array}\right]=\widehat{L}_{k}\left[\begin{array}{c}
I \\
\widehat{X}_{s}
\end{array}\right]\left(\widehat{X}_{s}^{-1} A^{T}\right)^{2^{k}},
$$


where

$$
\widehat{N}_{k}=\left[\begin{array}{cc}
\widehat{A}_{k} & 0 \\
\widehat{Q}_{k} & -I
\end{array}\right], \quad \widehat{L}_{k}=\left[\begin{array}{cc}
-\widehat{P}_{k} & I \\
\widehat{A}_{k}^{T} & 0
\end{array}\right]
$$

are defined by Algorithm 4.1, starting with $\widehat{A}_{0}=A^{T}, \widehat{Q}_{0}=Q, \widehat{P}_{0}=0$. It is easy to prove by induction that for all $k \geq 0$

$$
\widehat{A}_{k}=A_{k}^{T}, \quad \widehat{P}_{k}=Q-Q_{k}, \quad \widehat{Q}_{k}=Q-P_{k} .
$$

Indeed, assuming (4.7) for $k$, we have

$$
\widehat{Q}_{k+1}=\widehat{Q}_{k}-\widehat{A}_{k}^{T}\left(\widehat{Q}_{k}-\widehat{P}_{k}\right)^{-1} \widehat{A}_{k}=Q-P_{k}-A_{k}\left(Q_{k}-P_{k}\right)^{-1} A_{k}^{T}=Q-P_{k+1},
$$

and similarly we have $\widehat{A}_{k+1}=A_{k+1}^{T}$ and $\widehat{P}_{k+1}=Q-Q_{k+1}$.

By (4.6) and (4.7) we now have

$$
A_{k}^{T}=\left(\widehat{X}_{s}-\widehat{P}_{k}\right)\left(\widehat{X}_{s}^{-1} A^{T}\right)^{2^{k}}, \quad \widehat{Q}_{k}-\widehat{X}_{s}=A_{k}\left(\widehat{X}_{s}^{-1} A^{T}\right)^{2^{k}} .
$$

By (4.5), (4.8) and (4.7), we have

$$
\begin{aligned}
Q_{k}-X_{s} & =A_{k}^{T}\left(X_{s}^{-1} A\right)^{2^{k}} \\
& =\left(\widehat{X}_{s}-\widehat{P}_{k}\right)\left(\widehat{X}_{s}^{-1} A^{T}\right)^{2^{k}}\left(X_{s}^{-1} A\right)^{2^{k}} \\
& =\left(Q_{k}-X_{s}+\left(X_{s}+\widehat{X}_{s}-Q\right)\right)\left(\widehat{X}_{s}^{-1} A^{T}\right)^{2^{k}}\left(X_{s}^{-1} A\right)^{2^{k}},
\end{aligned}
$$

from which we obtain

$$
\left(Q_{k}-X_{s}\right)\left(I-\left(\widehat{X}_{s}^{-1} A^{T}\right)^{2^{k}}\left(X_{s}^{-1} A\right)^{2^{k}}\right)=\left(X_{s}+\widehat{X}_{s}-Q\right)\left(\widehat{X}_{s}^{-1} A^{T}\right)^{2^{k}}\left(X_{s}^{-1} A\right)^{2^{k}} .
$$

It follows that

$$
\limsup _{k \rightarrow \infty} \sqrt[2^{k}]{\left\|Q_{k}-X_{s}\right\|} \leq \rho\left(\widehat{X}_{s}^{-1} A^{T}\right) \rho\left(X_{s}^{-1} A\right)=\left(\rho\left(X_{s}^{-1} A\right)\right)^{2}<1 .
$$

So $Q_{k}$ converges to $X_{s}$ quadratically. Then we know $\left\{\widehat{P}_{k}\right\}$ is bounded and have by the first equation in (4.8) that

$$
\limsup _{k \rightarrow \infty} \sqrt[2^{k}]{\left\|A_{k}\right\|} \leq \rho\left(X_{s}^{-1} A\right)<1
$$

So $A_{k}$ converges to 0 quadratically. By the second equations in (4.7) and (4.8) we get

$$
\limsup _{k \rightarrow \infty} \sqrt[2^{k}]{\left\|\left(Q-P_{k}\right)-\widehat{X}_{s}\right\|} \leq\left(\rho\left(X_{s}^{-1} A\right)\right)^{2}<1 .
$$

So $Q-P_{k}$ converges to $\widehat{X}_{s}$ quadratically. This completes the proof of (b).

Algorithm 4.1 is said to be structure-preserving since for each $k \geq 0 N_{k}$ and $L_{k}$ have the structures given in (4.2) and the pencil $N_{k}-\lambda L_{k}$ is $T$-symplectic.

The complexity of Algorithm 4.1 can be reduced drastically by using the special structure of the matrix $A$ given by (2.6). Write $Q_{k}=Q-R_{k}$. Then it is easy to see by induction that the matrices $A_{k}, R_{k}$, and $P_{k}$ have the special forms

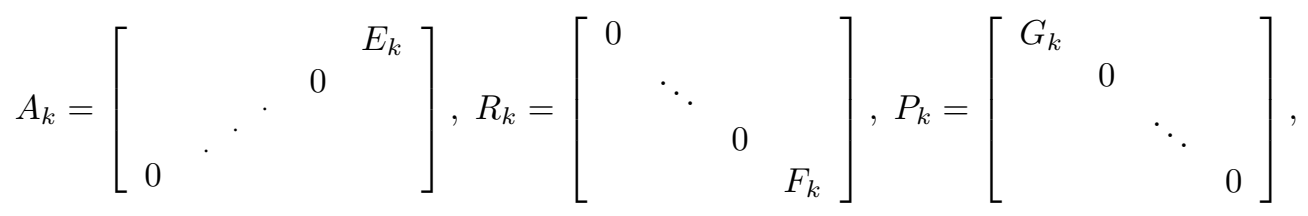


where the $q \times q$ matrices $E_{k}, F_{k}$, and $G_{k}$ can be determined by the following simplified algorithm, in which

$$
Q=\left[\begin{array}{cccc}
H_{0} & H_{1}^{T} & & \\
H_{1} & H_{0} & \ddots & \\
& \ddots & \ddots & H_{1}^{T} \\
& & H_{1} & H_{0}
\end{array}\right]_{m \times m}
$$

is given by $(2.5)$, with

$$
H_{0}=K_{0}+i \omega D_{0}-\omega^{2} M_{0}, \quad H_{1}=K_{1}+i \omega D_{1}-\omega^{2} M_{1} .
$$

Algorithm 4.2. Let $E_{0}=H_{1}, F_{0}=0, G_{0}=0$.

For $k=0,1, \ldots$, compute

$$
\begin{aligned}
& {\left[\begin{array}{c}
S_{k, 1} \\
S_{k, 2} \\
\vdots \\
S_{k, m}
\end{array}\right]=\left(Q-\left[\begin{array}{ccccc}
G_{k} & & & & \\
& 0 & & & \\
& & \ddots & & \\
& & & 0 & \\
& & & & F_{k}
\end{array}\right]\right)^{-1}\left[\begin{array}{c}
E_{k} \\
0 \\
\vdots \\
0
\end{array}\right],} \\
& \left.\left[\begin{array}{c}
T_{k, 1} \\
T_{k, 2} \\
\vdots \\
T_{k, m}
\end{array}\right]=\left(\begin{array}{ccccc}
G_{k} & & & \\
& 0 & & & \\
& & \ddots & & \\
& & & 0 & \\
& & & & F_{k}
\end{array}\right]\right)^{-1}\left[\begin{array}{c}
0 \\
\vdots \\
0 \\
E_{k}^{T}
\end{array}\right],
\end{aligned}
$$

where all matrix blocks on the left side of (4.11) and (4.12) are $q \times q$, and then compute

$$
E_{k+1}=E_{k} S_{k, m}, \quad F_{k+1}=F_{k}+E_{k}^{T} S_{k, 1}, \quad G_{k+1}=G_{k}+E_{k} T_{k, m} .
$$

The main task of Algorithm 4.2 is to solve the large sparse linear systems in (4.11) and (4.12). We rewrite the common matrix in (4.11) and (4.12) as

$$
Q-\left[\begin{array}{ccccc}
G_{k} & & & & \\
& 0 & & & \\
& & \ddots & & \\
& & & 0 & \\
& & & & F_{k}
\end{array}\right]=Q-B_{k} C_{k}^{T}
$$

with

$$
B_{k}=\left[\begin{array}{cccc}
G_{k}^{T} & 0 & \cdots & 0 \\
0 & \cdots & 0 & F_{k}^{T}
\end{array}\right]^{T}, \quad C_{k}^{T}=\left[\begin{array}{cccc}
I_{q} & 0 & \cdots & 0 \\
0 & \cdots & 0 & I_{q}
\end{array}\right],
$$

and solve the linear systems by the Sherman-Morrison-Woodbury formula

$$
\left(Q-B_{k} C_{k}^{T}\right)^{-1}=Q^{-1}+Q^{-1} B_{k}\left(I_{2 q}-C_{k}^{T} Q^{-1} B_{k}\right)^{-1} C_{k}^{T} Q^{-1} .
$$


Let $Q=U^{H} R$ be a qr-factorization of $Q$, where $U$ is unitary and $R$ is upper triangular. Since $Q^{T}=Q$, a linear system $Q X=B$ can be solved by

$$
X=R^{-1} U B \text { or } X=U^{T} R^{-T} B .
$$

Write $Q=\left[Q_{i j}\right]_{i, j=1}^{m}$ with

$$
\left\{\begin{array}{l}
Q_{i i}=H_{0}, \quad i=1, \cdots, m \\
Q_{i+1, i}=Q_{i, i+1}^{T}=H_{1}, \quad i=1, \cdots, m-1, \\
Q_{i j}=0_{q}, \quad|i-j|>1,
\end{array}\right.
$$

where $0_{q}$ is the $q \times q$ zero matrix. Let $\mathbb{U}_{k}$ denote the set of all $k \times k$ unitary matrices, and $\Delta_{k}$ the set of all $k \times k$ upper triangular matrices. The following algorithm computes the qr-factorization of $Q$ in a sparse way.

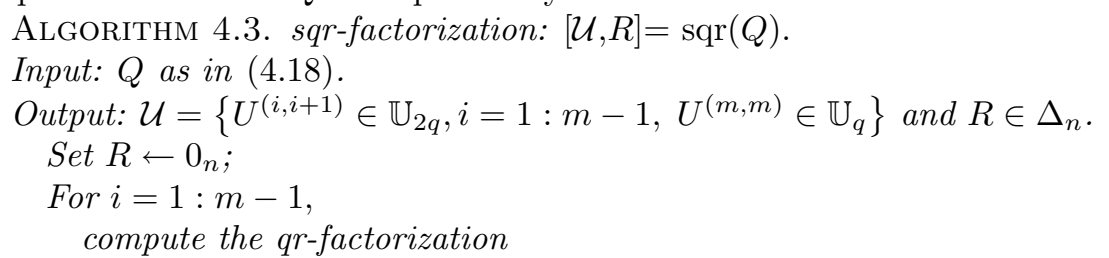

$$
\begin{aligned}
& {\left[\begin{array}{c}
Q_{i i} \\
Q_{i+1, i}
\end{array}\right] \rightarrow\left(U^{(i, i+1)}\right)^{H}\left[\begin{array}{c}
Q_{i i} \\
0
\end{array}\right],} \\
& \text { where } U^{(i, i+1)} \in \mathbb{U}_{2 q} \text { and the new } Q_{i i} \text { is in } \Delta_{q},
\end{aligned}
$$

compute

$$
\left[\begin{array}{c}
Q_{i j} \\
Q_{i+1, j}
\end{array}\right] \leftarrow U^{(i, i+1)}\left[\begin{array}{c}
Q_{i j} \\
Q_{i+1, j}
\end{array}\right], j=i+1: \min \{i+2, m\}
$$

Compute the qr-factorization $Q_{m m} \rightarrow\left(U^{(m, m)}\right)^{H} Q_{m, m}$,

where $U^{(m, m)} \in \mathbb{U}_{q}$ and the new $Q_{m m}$ is in $\Delta_{q}$;

$R_{i j} \leftarrow Q_{i j}, i=1: m, j=i: \min \{i+2, m\}$.

The above algorithm gives the qr-factorization $Q=U^{H} R$, where the unitary matrix $U=\left[U_{i, j}\right]_{i, j=1}^{m}$, with $U_{i, j} \in \mathbb{C}^{q \times q}$, is given in a sparse factored form. More precisely, $U=\widetilde{U}^{(m, m)} \prod_{i=m-1}^{1} \widetilde{U}^{(i, i+1)}$ with $\widetilde{U}^{(i, i+1)}$ and $\widetilde{U}^{(m, m)}$ being the extensions of $U^{(i, i+1)}$ and $U^{(m, m)}$, respectively, by adding appropriate 1 's on the diagonal. We now use the sqr-factorization of $Q$ to solve the linear system $Q X=B$ with $B=$ $\left[I_{q}, 0_{q}, \ldots, 0_{q}\right]^{T}$. Note that $I_{q}$ appears in the top position in $B$. In this process, $U_{i, 1}(i=1: m)$ are obtained explicitly for later use.

AlgORITHm 4.4. $\left[X_{1}, X_{m}, U_{1: m, 1}\right]=\operatorname{Sol}_{t}(\mathcal{U}, R)$.

Input: The output from Algorithm 4.3.

Output: The first and last submatrices of the solution $X=\left[X_{i}\right]_{i=1}^{m} \in \mathbb{C}^{n \times q}$ with $X_{i} \in \mathbb{C}^{q \times q}$ for the linear system $Q X=\left[I_{q}, 0_{q}, \ldots, 0_{q}\right]^{T}$, and the first block column of $U$.

Set $B_{1} \leftarrow I_{q}$;

For $i=1: m-1$, compute

$$
\left[\begin{array}{c}
B_{i} \\
B_{i+1}
\end{array}\right] \leftarrow U^{(i, i+1)}(1: 2 q, 1: q) B_{i}
$$

Compute $B_{m} \leftarrow U^{(m, m)} B_{m}$;

Set $U_{i, 1} \leftarrow B_{i}, i=1: m$;

For $i=m:-1: 1$, compute $X_{i}=R_{i i}^{-1}\left(B_{i}-\sum_{j=i+1}^{\min \{i+2, m\}} R_{i j} X_{j}\right)$.

For the linear system $Q X=B$ with $B=\left[0_{q}, \ldots, 0_{q}, I_{q}\right]^{T}$, it is possible to compute $X_{1}$ and $X_{m}$ directly without computing $X_{k}(k=2, \ldots, m-1)$. 
AlgORIthm 4.5. $\left[X_{1}, X_{m}\right]=\operatorname{Sol}_{b}\left(\mathcal{U}, R, U_{m, 1}\right)$.

Input: The output from Algorithm 4.3 and $U_{m, 1}$ from Algorithm 4.4.

Output: The first and the last submatrices of the solution $X=\left[X_{i}\right]_{i=1}^{m} \in \mathbb{C}^{n \times q}$

with $X_{i} \in \mathbb{C}^{q \times q}$ for the linear system $Q X=\left[0_{q}, \ldots, 0_{q}, I_{q}\right]^{T}$.

Set $\left[\begin{array}{c}B_{m-1} \\ B_{m}\end{array}\right] \leftarrow U^{(m-1, m)}(1: 2 q, q+1: 2 q)$;

Compute $B_{m} \leftarrow U^{(m, m)} B_{m} ; X_{m} \leftarrow R_{m, m}^{-1} B_{m} ;$ (by the 1st eq. in (4.17))

Compute $X_{1} \leftarrow U_{m, 1}^{T} R_{m, m}^{-T}$. (by the 2nd eq. in (4.17))

The following algorithm gives a more detailed implementation of Algorithm 4.2 and computes the stabilizing solutions of (3.2) and (3.4) by Theorem 4.1.

Algorithm 4.6. Computation of $X_{s}$ and $\widehat{X}_{s}$.

Input: $H_{0}, H_{1} \in \mathbb{C}^{q \times q}$, tolerance $\tau$.

Output: The solutions $X_{s} \in \mathbb{C}^{n \times n}$ for (3.2) and $\widehat{X}_{s} \in \mathbb{C}^{n \times n}$ for (3.4).

Take $Q$ in (4.18), $E_{0}=H_{1}, F_{0}=0, G_{0}=0$;

Call $[\mathcal{U}, R]=\operatorname{sqr}(Q)$

$\left[Y_{1}, Y_{m}, U_{1: m, 1}\right]=\operatorname{Sol}_{t}(\mathcal{U}, R)$

$\left[Z_{1}, Z_{m}\right]=\operatorname{Sol}_{b}\left(\mathcal{U}, R, U_{m, 1}\right)$

For $k=0,1, \cdots$

$X_{k, 1}=\left[Y_{1} G_{k}, Z_{1} F_{k}\right], X_{k, m}=\left[Y_{m} G_{k}, Z_{m} F_{k}\right] ;$

$\left[X_{k, 1}^{f}, X_{k, m}^{f}\right]=\left[Y_{1} E_{k}, Y_{m} E_{k}\right]$

$\left[X_{k, 1}^{g}, X_{k, m}^{g}\right]=\left[Z_{1} E_{k}^{T}, Z_{m} E_{k}^{T}\right]$;

$S_{k, i}=X_{k, i}^{f}+X_{k, i}\left[I_{2 q}-\left(\begin{array}{c}X_{k, 1} \\ X_{k, m}\end{array}\right)\right]^{-1}\left(\begin{array}{c}X_{k, 1}^{f} \\ X_{k, m}^{f}\end{array}\right), \quad i=1, m$,

$T_{k, m}=X_{k, m}^{g}+X_{k, m}\left[I_{2 q}-\left(\begin{array}{c}X_{k, 1} \\ X_{k, m}\end{array}\right)\right]^{-1}\left(\begin{array}{c}X_{k, 1}^{g} \\ X_{k, m}^{g}\end{array}\right)$;

$E_{k+1}=E_{k} S_{k, m}, F_{k+1}=F_{k}+E_{k}^{T} S_{k, 1}, G_{k+1}=G_{k}+E_{k} T_{k, m} ;$

If $\left\|E_{k}^{T} S_{k, 1}\right\| \leq \tau\left\|F_{k}\right\|$ and $\left\|E_{k} T_{k, m}\right\| \leq \tau\left\|G_{k}\right\|$, then

$X_{s} \leftarrow Q, X_{s}\left(n^{\prime}: n, n^{\prime}: n\right) \leftarrow H_{0}-F_{k+1}$,

$\widehat{X}_{s} \leftarrow Q, \widehat{X}_{s}(1: q, 1: q) \leftarrow H_{0}-G_{k+1}$,

where $n^{\prime}=(m-1) q+1$, and stop.

5. Numerical results. The sqr-factorization in Algorithm 4.3 requires about $\frac{86}{3} m q^{3}$ flops. The linear system solvers in Algorithm 4.4 and Algorithm 4.5 require $9 m q^{3}$ and $4 q^{3}$ flops, respectively. Each iteration of the doubling algorithm in Algorithm 4.6 requires about $\frac{154}{3} q^{3}$ flops. Algorithm 4.6 is efficient since no more than 10 iterations are typically needed for convergence. For large $q$ and $m$ the total computational work for Algorithm 4.6 is thus roughly $\frac{113}{3} m q^{3}$, assuming that the number of iterations for the doubling algorithm is bounded independent of $q$ and $m$. We note that Algorithms 4.3, 4.4 and 4.5 presented in this paper can also be used in the initial deflation procedure [5] for the linearization approach. So the deflation procedure can be completed in about $\frac{113}{3} m q^{3}$ flops as well.

In this section we present numerical results to illustrate the efficiency and accuracy of the solvent approach for computing the eigenpairs of the QEP (2.3), through computing the solvent $X_{s}$ by Algorithm 4.6.

We first explain how the eigenpairs can be computed after the solvent $X_{s}$ is obtained. By Algorithm 4.6 we see that

$$
Q-X_{s}=\left[0, \cdots, 0, I_{q}\right]^{T}\left[0, \cdots, 0, F_{\infty}\right]
$$


where $F_{\infty}=\lim _{k \rightarrow \infty} F_{k}$. Write $A=\left[\begin{array}{cc}0_{n-q} & H_{1}^{t} \\ 0 & 0_{q}\end{array}\right]$, where $H_{1}^{t}=\left[H_{1}^{T}, 0, \cdots, 0\right]^{T}$. Applying $U$ (given implicitly in a sparse factored form) in Algorithm 4.3 to $A$ and $X_{s}$, respectively, we have

$$
U A=\left[\begin{array}{cc}
0_{n-q} & \widetilde{H}_{1}^{t} \\
0 & \Phi_{1}
\end{array}\right], U X_{s}=\left[\begin{array}{cc}
X_{1} & X_{2} \\
0 & \Phi_{2}
\end{array}\right]
$$

where $X_{1}=R(1: n-q, 1: n-q)$ and $X_{2}(1: n-3 q, 1: q)=0$. From the factorization $P(\lambda)=\left(\lambda A^{T}+X_{s}\right) X_{s}^{-1}\left(\lambda X_{s}+A\right)$, the nonzero stable eigenpairs $\left(\lambda_{s}, z_{s}\right)$ of $P(\lambda)$ are those of $\lambda X_{s}+A$ and can be computed by

$$
\begin{gathered}
\Phi_{1} z_{s, 2}=-\lambda_{s} \Phi_{2} z_{s, 2}, \\
z_{s, 1}=-X_{1}^{-1}\left(X_{2} z_{s, 2}+\lambda_{s}^{-1} \widetilde{H}_{1}^{t} z_{s, 2}\right), z_{s}=\left[\begin{array}{c}
z_{s, 1} \\
z_{s, 2}
\end{array}\right],
\end{gathered}
$$

for $s=1, \ldots, q$. Recall that the first block column of $U$ is known from Algorithm 4.4. So $\Phi_{1}=U_{m, 1} H_{1}$ and $\widetilde{H}_{1}^{t}=U_{1: m-1,1} H_{1}$.

If we are only interested in the eigenvalues, then we can find all nonzero stable eigenvalues from (5.3) and get all finite unstable eigenvalues by taking the reciprocals of the nonzero stable ones. The cost is $O\left(q^{3}\right)$ flops. Eigenvectors corresponding to nonzero stable eigenvalues can be found from (5.4) with a cost of $7 \mathrm{mq}^{3}$ flops, noting that $X_{1}$ is block 3-banded upper triangular and that $\widetilde{H}_{1}^{t} z_{s, 2}=U_{1: m-1,1}\left(H_{1} z_{s, 2}\right)$.

Some further work is required if the eigenvectors corresponding to finite unstable eigenvalues are also needed. We first compute all left eigenvectors of $\lambda \Phi_{2}+\Phi_{1}$ by

$$
y_{s}^{T} \Phi_{1}=-\lambda_{s} y_{s}^{T} \Phi_{2}
$$

for $s=1, \cdots, q$, at a cost of $O\left(q^{3}\right)$ flops. The finite unstable eigenpairs $\left(\lambda_{u}, z_{u}\right)$ of $P(\lambda)$ satisfy

$$
P\left(\lambda_{u}\right) z_{u} \equiv P\left(1 / \lambda_{s}\right) z_{u}=\frac{1}{\lambda_{s}^{2}}\left(A^{T}+\lambda_{s} X_{s}\right) X_{s}^{-1}\left(X_{s}+\lambda_{s} A\right) z_{u}=0 .
$$

From (5.2) and (5.5) follows that

$$
\left(A^{T}+\lambda_{s} X_{s}\right) U^{T}\left[\begin{array}{c}
0 \\
y_{s}
\end{array}\right]=\left(\left[\begin{array}{cc}
0 & 0 \\
\widetilde{H}_{1} & \Phi_{1}^{T}
\end{array}\right]+\left[\begin{array}{cc}
\lambda_{s} X_{1}^{T} & 0 \\
\lambda_{s} X_{2}^{T} & \lambda_{s} \Phi_{2}^{T}
\end{array}\right]\right)\left[\begin{array}{c}
0 \\
y_{s}
\end{array}\right]=0 .
$$

From (5.6) and (5.2) the eigenvector $z_{u}$ corresponding to $\lambda_{u}=\lambda_{s}^{-1}$ can be found by solving the linear system

$$
\left(X_{s}+\lambda_{s} A\right) z_{u}=X_{s}\left(U^{T}\left[\begin{array}{c}
0 \\
y_{s}
\end{array}\right]\right)=\left[\begin{array}{c}
0 \\
\Phi_{2}^{T} y_{s}
\end{array}\right] .
$$

Pre-multiplying (5.8) with $U$ and using (5.2) again, we see that the finite unstable eigenpairs $\left(\lambda_{u}, z_{u}\right)$ of $P(\lambda)$ can be computed by

$$
\left[\begin{array}{l}
\zeta_{u, 1} \\
\zeta_{u, 2}
\end{array}\right]=U\left[\begin{array}{c}
0 \\
\Phi_{2}^{T} y_{s}
\end{array}\right], \quad z_{u, 2}=\left(\Phi_{2}+\lambda_{s} \Phi_{1}\right)^{-1} \zeta_{u, 2}
$$




$$
z_{u, 1}=X_{1}^{-1}\left[\zeta_{u, 1}-\left(X_{2}+\lambda_{s} \widetilde{H}_{1}^{t}\right) z_{u, 2}\right], \quad z_{u}=\left[\begin{array}{c}
z_{u, 1} \\
z_{u, 2}
\end{array}\right]
$$

for $u=1, \cdots, q$. Note that $\Phi_{2}+\lambda_{s} \Phi_{1}$ is nonsingular since $\Phi_{2}+\lambda \Phi_{1}$ only has unstable eigenvalues by (5.3). The vectors $z_{u, 2}$ in (5.9) can be found in $O\left(q^{3}\right)$ flops via a Hessenberg-triangular form of the pair $\left(\Phi_{2}, \Phi_{1}\right)$ obtained by the qz-algorithm. So (5.9) requires $O\left(q^{3}\right)$ flops, while $(5.10)$ requires $7 m q^{3}$ flops.

\begin{tabular}{|c|c|c|c|c|}
\hline & $\omega=100$ & $\omega=1000$ & $\omega=3000$ & $\omega=5000$ \\
\hline$k$ & $\rho=0.9622$ & $\rho=0.8831$ & $\rho=0.8080$ & $\rho=0.7569$ \\
\hline 1 & $2.0 e-02$ & $1.4 e-02$ & $9.7 e-03$ & $8.1 e-03$ \\
\hline 2 & $3.4 e-03$ & $1.8 e-03$ & $1.7 e-03$ & $1.5 e-03$ \\
\hline 3 & $7.4 e-04$ & $6.0 e-04$ & $2.9 e-04$ & $1.5 e-04$ \\
\hline 4 & $3.2 e-04$ & $8.2 e-05$ & $9.3 e-06$ & $1.8 e-06$ \\
\hline 5 & $1.0 e-04$ & $1.6 e-06$ & $9.3 e-09$ & $2.4 e-10$ \\
\hline 6 & $8.5 e-06$ & $5.4 e-10$ & $9.5 e-15$ & $2.3 e-18$ \\
\hline 7 & $6.1 e-08$ & $6.2 e-17$ & 0 & \\
\hline 8 & $3.2 e-12$ & 0 & & \\
\hline 9 & $1.1 e-22$ & & & \\
\hline 10 & 0 & & & \\
\hline
\end{tabular}

Table 5.1. $\left\|F_{k+1}-F_{k}\right\|_{2} /\left\|F_{k}\right\|_{2}$ for different $\omega$ values, $(q, m)=(159,11)$

\begin{tabular}{|c|c|c|c|c|}
\hline & $\omega=100$ & $\omega=1000$ & $\omega=3000$ & $\omega=5000$ \\
\hline$k$ & $\rho=0.9307$ & $\rho=0.7933$ & $\rho=0.6692$ & $\rho=0.5953$ \\
\hline 1 & $2.2 e-02$ & $1.3 e-02$ & $1.1 e-02$ & $8.4 e-03$ \\
\hline 2 & $3.9 e-03$ & $1.9 e-03$ & $9.3 e-04$ & $5.9 e-04$ \\
\hline 3 & $1.2 e-03$ & $2.4 e-04$ & $3.8 e-05$ & $9.5 e-06$ \\
\hline 4 & $2.3 e-04$ & $6.0 e-06$ & $6.2 e-08$ & $2.4 e-09$ \\
\hline 5 & $2.3 e-05$ & $3.6 e-09$ & $1.6 e-13$ & $1.4 e-16$ \\
\hline 6 & $2.3 e-07$ & $1.3 e-15$ & 0 & 0 \\
\hline 7 & $2.4 e-11$ & 0 & & \\
\hline 8 & $1.3 e-20$ & & & \\
\hline 9 & 0 & & & \\
\hline
\end{tabular}

Table 5.2. $\left\|F_{k+1}-F_{k}\right\|_{2} /\left\|F_{k}\right\|_{2}$ for different $\omega$ values, $(q, m)=(303,19)$

In the linearization approach, the computation of stable and unstable eigenvectors involves the successive application of the inverses of the two potentially ill-conditioned matrices used in the initial deflation process [5]. In our solvent approach, the matrix $X_{s}$ used in the computation of stable eigenpairs is usually well-conditioned. So we expect to have better accuracy in the computed results, at least for stable eigenpairs, when using the solvent approach proposed in this paper.

We now present numerical results on three sets of test data generated by a finite element package, with $(q, m)=(159,11),(303,19),(705,51)$, respectively. The matrices $M$ and $K$ are given by (2.1) and (2.2), and we take $D=0.8 M+0.2 K$. All numerical experiments are carried out in MATLAB 2008b with machine precision eps $\approx 2.22 \times 10^{-16}$.

Our solvent approach is efficient since we have fully exploited the sparse structure in the QEP. The only uncertainty is the number of iterations needed for the 


\begin{tabular}{|c|c|c|c|c|}
\hline & $\omega=100$ & $\omega=1000$ & $\omega=3000$ & $\omega=5000$ \\
\hline$k$ & $\rho=0.9593$ & $\rho=0.8745$ & $\rho=0.7925$ & $\rho=0.7406$ \\
\hline 1 & $1.1 e-01$ & $1.0 e-01$ & $7.0 e-02$ & $5.7 e-02$ \\
\hline 2 & $2.8 e-02$ & $1.2 e-02$ & $1.0 e-02$ & $8.8 e-03$ \\
\hline 3 & $4.7 e-03$ & $3.6 e-03$ & $1.5 e-03$ & $7.8 e-04$ \\
\hline 4 & $2.1 e-03$ & $4.2 e-04$ & $3.8 e-05$ & $6.4 e-06$ \\
\hline 5 & $5.7 e-04$ & $5.8 e-06$ & $2.2 e-08$ & $4.3 e-10$ \\
\hline 6 & $4.0 e-05$ & $1.1 e-09$ & $7.7 e-15$ & $2.9 e-19$ \\
\hline 7 & $1.9 e-07$ & $3.5 e-17$ & 0 & \\
\hline 8 & $4.6 e-12$ & 0 & & \\
\hline 9 & 0 & & & \\
\hline
\end{tabular}

Table 5.3. $\left\|F_{k+1}-F_{k}\right\|_{2} /\left\|F_{k}\right\|_{2}$ for different $\omega$ values, $(q, m)=(705,51)$

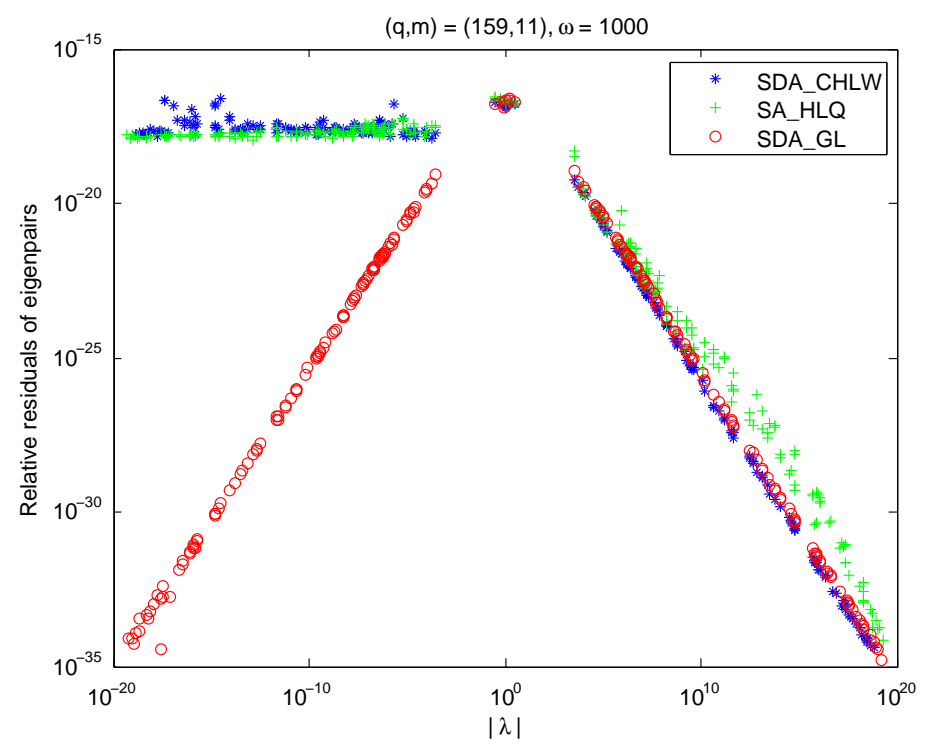

convergence of $\left\{F_{k}\right\}$ and $\left\{G_{k}\right\}$ in Algorithm 4.6. In Tables 5.1-5.3 we give $\| F_{k+1}-$ $F_{k}\left\|_{2} /\right\| F_{k} \|_{2}$ for the three pairs of $(q, m)$ and for $\omega=100,1000,3000,5000$, respectively. The values $\rho=\rho\left(X_{s}^{-1} A\right)$ are also given for the $\omega$ values. From the tables we can see that the sequence $\left\{F_{k}\right\}$ converges within 10 iterations for each $\omega$. The convergence behaviour of $\left\{G_{k}\right\}$ is roughly the same, as indicated by Theorem 4.1. There is no significant difference in the performance of Algorithm 4.6 for different values of $(q, m)$.

To show numerically that our method has better accuracy than existing methods, we compare our method (SDA_GL) to the method in [5] (SDA_CHLW) and the method SA-I in [16] (SA_HLQ). The latter method has been shown in [16] to have better accuracy than two other methods compared there.

To measure the accuracy of an approximate eigenpair $(\lambda, z)$ for $P(\lambda)$ we use the relative residual

$$
\text { RRes }=\frac{\left\|\lambda^{2} A^{T} z+\lambda Q z+A z\right\|_{2}}{\left(|\lambda|^{2}\|A\|_{F}+|\lambda|\|Q\|_{F}+\|A\|_{F}\right)\|z\|_{2}} .
$$

In the three figures we plot for $\omega=1000$ the relative residuals of approximate eigen- 

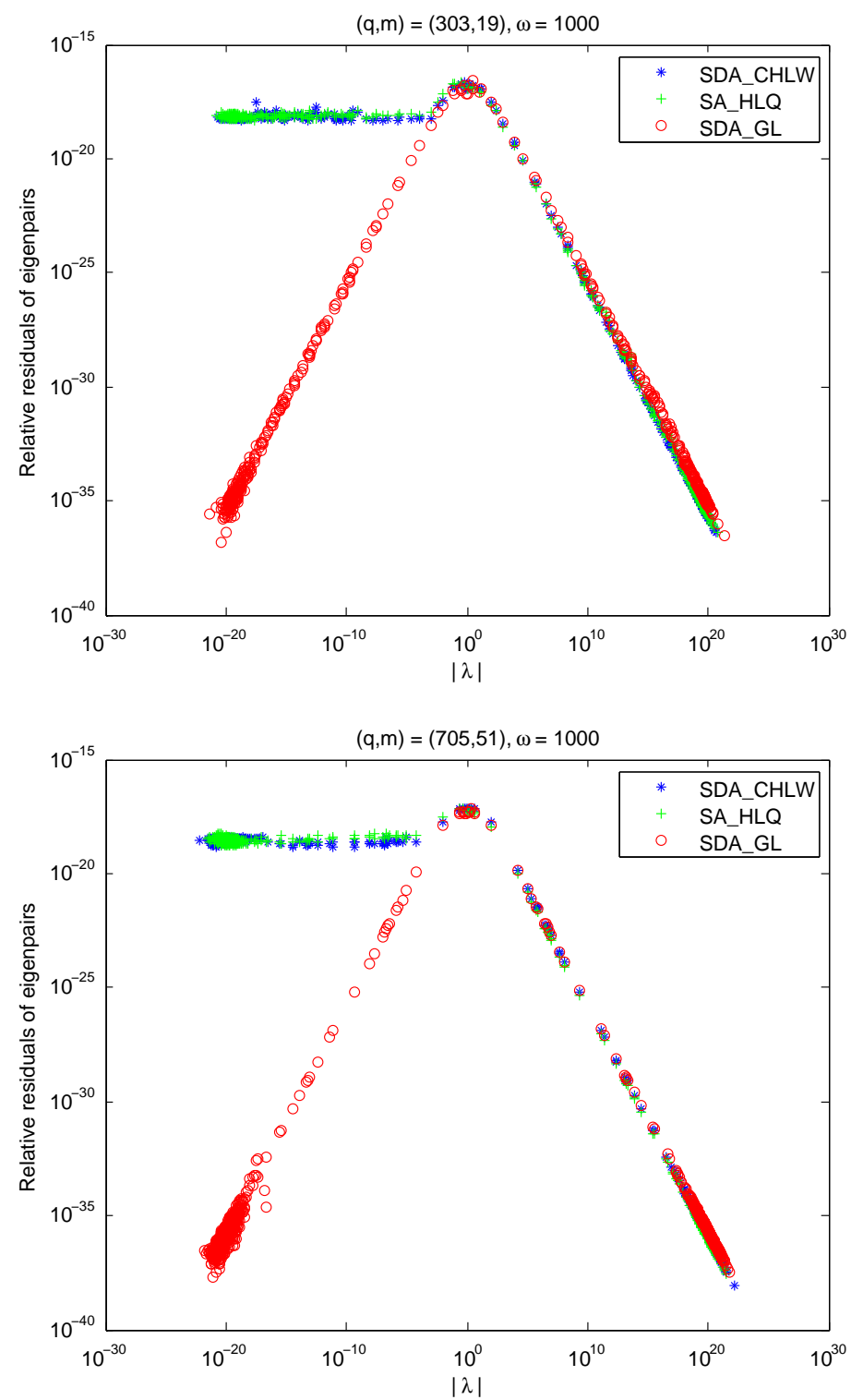

pairs, for $(q, m)=(159,11),(303,19),(705,51)$, respectively. Indeed, our new method (SDA_GL) has significantly better accuracy for stable eigenpairs.

6. Conclusion. We have solved a structured quadratic eigenvalue problem efficiently and accurately, by using a structure-preserving doubling algorithm in the solvent approach. The doubling algorithm has fast convergence and exploits the sparsity of the QEP. Theoretical issues involved in this solvent approach are settled satisfactorily. In particular, we present a generalization of the classical Bendixson's theorem to bounded linear operators in infinite-dimensional Hilbert spaces, which could also be useful elsewhere. We also mention that the solvent approach studied in this paper can also be applied to QEPs with more general sparsity structures, such as the QEPs arising in SAW-filter simulations [29]. 
Acknowledgments. We thank Dr. Chin-Tien Wu from National Chiao Tung University for discussions about the finite element model that leads to the quadratic eigenvalue problem and for generating the test data used in our numerical experiments. We are also grateful to two referees for their very helpful comments.

\section{REFERENCES}

[1] A. G. BASKakov, Dichotomy of the spectrum of non-self-adjoint operators, (Russian) Sibirsk. Mat. Zh., 32 (3) (1991), pp. 24-30; translation in Siberian Math. J., 32 (3) (1992), pp. $370-375$.

[2] I. Bendixson, Sur les racines d'une équation fondamentale, (French) Acta Math., 25 (1902), pp. 359-365.

[3] D. A. Bini, L. Gemignani, and B. Meini, Computations with infinite Toeplitz matrices and polynomials, Linear Algebra Appl., 343-344 (2002), pp. 21-61.

[4] C.-Y. Chiang, E. K.-W. Chu, C.-H. Guo, T.-M. Huang, W.-W. Lin, and S.-F. Xu, Convergence analysis of the doubling algorithm for several nonlinear matrix equations in the critical case, SIAM J. Matrix Anal. Appl., 31 (2009), pp. 227-247.

[5] E. K.-W. Chu, T.-M. Hwang, W.-W. Lin, And C.-T. Wu, Vibration of fast trains, palindromic eigenvalue problems and structure-preserving doubling algorithms, J. Comput. Appl. Math., 219 (2008), pp. 237-252.

[6] G. J. DAvIS, Numerical solution of a quadratic matrix equation, SIAM J. Sci. Stat. Comput., 2 (1981), pp. 164-175.

[7] J. C. Engwerda, A. C. M. Ran, And A. L. Rijkeboer, Necessary and sufficient conditions for the existence of a positive definite solution of the matrix equation $X+A^{*} X^{-1} A=Q$, Linear Algebra Appl., 186 (1993), pp. 255-275.

[8] I. Gohberg, S. Goldberg, and M. A. KaAshoek, Classes of Linear Operators, Vol. II, Operator Theory: Advances and Applications, Vol. 63, Birkhäuser, Basel, 1993.

[9] C.-H. Guo, Numerical solution of a quadratic eigenvalue problem, Linear Algebra Appl., 385 (2004), pp. 391-406.

[10] C.-H. Guo and P. Lancaster, Iterative solution of two matrix equations, Math. Comp., 68 (1999), pp. 1589-1603.

[11] C.-H. Guo and P. Lancaster, Algorithms for hyperbolic quadratic eigenvalue problems, Math. Comp., 74 (2005), pp. 1777-1791.

[12] U. Haagerup and S. Thorbjørnsen, A new application of random matrices: $\operatorname{Ext}\left(C_{\text {red }}^{*}\left(F_{2}\right)\right)$ is not a group, Ann. of Math., 162 (2005), pp. 711-775.

[13] N. J. Higham AND H.-M. Kim, Numerical analysis of a quadratic matrix equation, IMA J. Numer. Anal., 20 (2000), pp. 499-519.

[14] A. Hilliges, Numerische Lösung von quadratischen eigenwertproblemen mit Anwendung in der Schienendynamik, Master's thesis, Technical University Berlin, Berlin, Germany, 2004.

[15] A. Hilliges, C. Mehl, and V. Mehrmann, On the solution of palindromic eigenvalue problems, in Proceedings of the 4th European Congress on Computational Methods in Applied Sciences and Engineering (ECCOMAS), Jyväskylä, Finland, 2004.

[16] T.-M. HuAng, W.-W. Lin, AND J. QIAN, Structure-preserving algorithms for palindromic quadratic eigenvalue problems arising from vibration of fast trains, SIAM J. Matrix Anal. Appl., 30 (2009), pp. 1566-1592.

[17] I. C. F. IPSEn, Accurate eigenvalues for fast trains, SIAM News, 37 (9) (2004), pp. 1-2.

[18] D. Kressner, C. SchröDer, And D. S. Watkins, Implicit QR algorithms for palindromic and even eigenvalue problems, Numer. Algorithms, 51 (2009), pp. 209-238.

[19] P. Lancaster, U. Prells, and L. Rodman, Canonical structures for palindromic matrix polynomials, Operators and Matrices, 1 (2007), pp. 469-489.

[20] R.-C. LI, W.-W. Lin, AND C.-S. WANG, Structured backward error for palindromic polynomial eigenvalue problems, Numer. Math., 116 (2010), pp. 95-122.

[21] W.-W. Lin AND S.-F. XU, Convergence analysis of structure-preserving doubling algorithms for Riccati-type matrix equations, SIAM J. Matrix Anal. Appl., 28 (2006), pp. 26-39.

[22] D. S. Mackey, N. Mackey, C. Mehl, and V. Mehrmann, Structured polynomial eigenvalue problems: good vibrations from good linearizations, SIAM J. Matrix Anal. Appl., 28 (2006), pp. 1029-1051.

[23] D. S. Mackey, N. Mackey, C. Mehl, and V. Mehrmann, Numerical methods for palindromic eigenvalue problems: Computing the anti-triangular Schur form, Numer. Linear Algebra Appl., 16 (2009), pp. 63-86. 
[24] C. van der Mee, G. Rodriguez, and S. Seatzu, LDU Factorization results for bi-infinite and semi-infinite scalar and block Toeplitz matrices, Calcolo, 33 (1996), pp. 307-335.

[25] B. Meini, Efficient computation of the extreme solutions of $X+A^{*} X^{-1} A=Q$ and $X-$ $A^{*} X^{-1} A=Q$, Math. Comp., 71 (2002), pp. 1189-1204.

[26] F. Tisseur and K. Meerbergen, The quadratic eigenvalue problem, SIAM Rev., 43 (2001), pp. 235-286.

[27] J. Weidmann, Linear Operators in Hilbert Spaces, Graduate Texts in Mathematics, 68, Springer-Verlag, New York, 1980.

[28] H. Wielandt, On eigenvalues of sums of normal matrices, Pacific J. Math., 5 (1955), pp. 633-638.

[29] S. ZaglmaYr, Eigenvalue problems in SAW-filter simulations, Master's thesis, Johannes Kepler University, Linz, Austria, September 2002.

[30] X. ZHAN, Computing the extremal positive definite solutions of a matrix equation, SIAM J. Sci. Comput., 17 (1996), pp. 1167-1174. 